\title{
Comportamiento de los tallos no cementados recubiertos con hidroxiapatita colocados con alineación coronal inadecuada en la artroplastia primaria de cadera. Resultados del seguimiento a largo plazo
}

\author{
Harold Simesen de Bielke," Leonel Pérez Alamino," Germán Garabano,"* César Á. Pesciallo," \\ Hernán del Sel," Fernando A. Lopreite** \\ "Servicio de Ortopedia y Traumatología, Sanatorio Modelo S.A., San Miguel de Tucumán, Tucumán, Argentina \\ ${ }^{*}$ Servicio de Ortopedia y Traumatología, Hospital Británico de Buenos Aires, Ciudad Autónoma de Buenos Aires, Argentina
}

\begin{abstract}
RESUMEN
Introducción: La mala alineación de los tallos femorales no cementados sigue siendo un tema controvertido. Algunos autores han comunicado una inadecuada osteointegración y hundimiento en los tallos en varo y otros no encontraron dichos efectos adversos, pese a la incorrecta alineación. Los objetivos de este estudio fueron determinar la prevalencia de tallos no cementados con cobertura total de hidroxiapatita en deseje coronal y mostrar los resultados clínico-radiográficos a largo plazo. Materiales y Métodos: Estudio retrospectivo entre 2006 y 2009. Se analizaron 220 reemplazos totales de cadera primarios con tallos no cementados con cobertura completa de hidroxiapatita. La alineación coronal fue analizada con radiografías anteroposteriores de ambas caderas en $10^{\circ}$ de rotación interna. Se registraron datos, como osteointegración, hundimiento, osteólisis femoral proximal e hipertrofia cortical. Para el análisis clínico se utilizó el Harris Hip Score. El análisis de supervivencia contempló la necesidad de revisión por cualquier causa. Resultados: La prevalencia de tallos en deseje fue del $32,3 \%$, con mayoría de implantes en varo (73,3\%). El Harris Hip Score tuvo un incremento estadísticamente significativo $(41,05 \pm 6,5$ vs. $90,05 \pm 2,5 ; p<0,01)$. El $73,1 \%$ de los tallos desarrollaron una fijación ósea estable. Se observó osteólisis femoral proximal en el $3 \%$ de los casos. Hubo un $3 \%$ de fracturas periprotésicas femorales. La supervivencia de la prótesis fue del $100 \%$ a los 10.9 años. Conclusión: Los tallos no cementados con cobertura total de hidroxiapatita toleran adecuadamente la alineación coronal en varo/valgo a largo plazo.

Palabras clave: Artroplastia total de cadera; tallos no cementados; deseje coronal; revisión de cadera.
\end{abstract}

Nivel de Evidencia: IV

Behavior of Hydroxyapatite-Coated Cementless Stems Placed With Coronal Malalignment in Primary Hip Arthroplasty. Long-Term Follow-Up Results

\section{ABSTRACT}

Introduction: Coronal malalignment of non-cemented stems remains controversial. It's been reported that femoral stems implanted with varus or valgus developed subsidence and lack of integration with femoral bone. The purpose of this study was to calculate prevalence of coronal malalignment in cementless, fully coated with hydroxialapatyte (HA) femoral stems focusing in their longterm clinical and radiographic outcomes. Materials and Methods: A retrospective study was performed, assessing 220 primaries total hip replacements with non-cemented, fully coated with HA, femoral stems between 2006 and 2009. Coronal alignment was assessed with hips antero-posterior views with $10^{\circ}$ of internal rotation of lower limbs. We registered data about subsidence, proximal femoral osteolysis and cortical hipertrophy. Functional outcomes were assessed with Harris Hip Score (HHS). Free revision rate implant survival was calculated. Results: Prevalence of femoral stems with malalignment was $32.3 \%$. HHS showed an statistically significant increase after surgery $(41.05 \pm 6.5$ versus $90.05 \pm 2.5 ; p<0.01)$. There were $73.1 \%$ of femoral stems that achieved a bone stable fixation. We observed proximal femoral osteolysis in $3.0 \%$ of the patients and there were $3 \%$ of periprosthetic fractures. Free-revision survival rate was $100 \%$ at 10.9 years of follow-up. Conclusion: Cementless, fully coated with HA femoral stems with varus/valgus malalignment achieves good long-term clinical and radiographic outcomes.

Keywords: Total hip arthroplasty; cementless femoral stems; coronal malalignment; hip revision.

Level of Evidence: IV

Recibido el 17-2-2021. Aceptado luego de la evaluación el 16-3-2021 • Dr. HAROLD SIMESEN DE BIELKE • haroldsimesen @ hotmail.com https://orcid.org/0000-0003-2922-1440 Cómo citar este artículo: Simesen de Bielke H, Pérez Alamino L, Garabano G, Pesciallo CÁ, del Sel H, Lopreite FA. Comportamiento de los tallos no cementados recubiertos con hidroxiapatita colocados con alineación coronal inadecuada en la artroplastia primaria de cadera. Resultados del seguimiento a largo plazo. Rev Asoc Argent Ortop Traumatol 2021;86(4):455-462. https://doi.org/10.15417/issn. 1852-7434.2021.86.4.1321 


\section{INTRODUCCIÓN}

El incorrecto posicionamiento de los componentes protésicos constituye una potencial complicación en el reemplazo total de cadera (RTC). ${ }^{1}$ Se sabe que, para obtener un resultado óptimo en el RTC cementado, el sitio de entrada en el canal femoral debe ser preciso y la colocación en los ejes coronal y sagital debe ser la adecuada, con el objetivo de evitar un espesor heterogéneo del manto de cemento alrededor del tallo. ${ }^{2}$ Clásicamente, la colocación en varo del componente femoral cementado se asocia a fractura del manto de cemento, hundimiento y aflojamiento mecánico precoz. ${ }^{3,4}$ En cuanto a los tallos no cementados, los efectos de la mala alineación siguen siendo aún controvertidos. Las probables complicaciones estarían representadas por la mayor incidencia de fracturas intraoperatorias, una fijación inestable, el aflojamiento precoz y el dolor de muslo posoperatorio. ${ }^{4-7}$ Algunos autores han publicado una osteointegración subóptima e inestabilidad por subdimensionamiento del implante en los tallos no cementados colocados en varo, debido al inadecuado asentamiento del componente femoral en el fémur proximal. ${ }^{8-10}$ Sin embargo, otros autores no encontraron dichos efectos adversos, y obtuvieron resultados alentadores, pese al inadecuado posicionamiento femoral. ${ }^{5,11}$

Los objetivos de este estudio fueron determinar la prevalencia de tallos no cementados con cobertura completa de hidroxiapatita implantados en una posición inadecuada, en el eje coronal, haciendo énfasis en los resultados clínico-radiográficos y establecer la supervivencia de la prótesis a largo plazo en este grupo de pacientes.

\section{MATERIALES Y MÉTODOS}

Según un análisis retrospectivo de la base de datos de nuestro Servicio, se realizaron 681 RTC primarios entre 2006 y 2009. En 255 (37,4\%) RTC, se utilizó un componente femoral no cementado; 220 (32,3\%) casos correspondían exclusivamente a tallos recubiertos de hidroxiapatita en forma completa (Corail, Depuy Synthes, Warsaw, Ind., EE.UU.) (Figura 1).

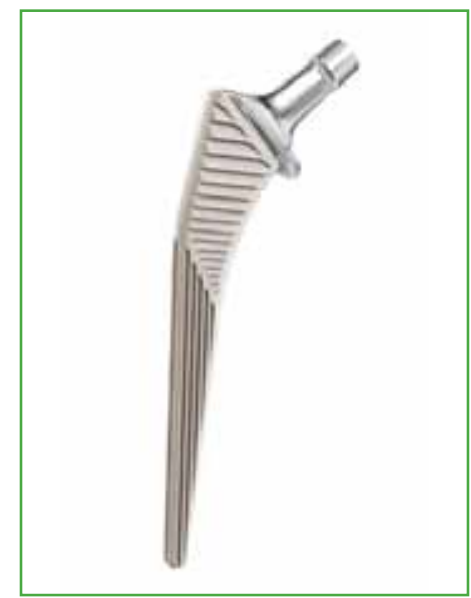

Figura 1. Tallo Corail® (fijación no cementada).

Alma de titanio con total cobertura de hidroxiapatita.

Modelo sin apoyo en el calcar.

Para determinar los tallos con inadecuada alineación coronal, dos observadores efectuaron un análisis radiográfico, de forma independiente, de los 220 RTC mencionados. Se utilizó la radiografía anteroposterior de ambas caderas con $10^{\circ}$ de rotación interna, de ambos miembros inferiores, tomada en el posoperatorio inmediato. Se midió el ángulo formado entre el eje longitudinal del tallo y el de la diáfisis femoral, y se consideró deseje (en varo o en valgo) si el ángulo era $\geq 2^{\circ}$.

Los criterios de inclusión fueron: RTC primario, utilización de un tallo no cementado con recubrimiento completo de hidroxiapatita (Corail®), posicionamiento inadecuado en el plano coronal y un seguimiento mínimo de 10 años. Los diagnósticos que motivaron las artroplastias se detallan en la Tabla 1. 
Tabla 1. Diagnósticos preoperatorios de la serie evaluada

\begin{tabular}{|l|c|}
\hline Diagnósticos & n $(\%)$ \\
\hline Coxartrosis idiopática & $58(86,5 \%)$ \\
\hline Osteonecrosis & $5(7,5 \%)$ \\
\hline Coxartrosis por displasia del desarrollo de la cadera & $3(4,5 \%)$ \\
\hline Coxartrosis por epifisiólisis & $1(1,5 \%)$ \\
\hline
\end{tabular}

\section{Técnica quirúrgica}

Todos los pacientes fueron operados por el mismo equipo quirúrgico, en quirófano de flujo laminar, bajo anestesia raquídea hipotensiva. Siempre el abordaje fue en decúbito dorsal, por vía lateral directa modificada. ${ }^{12} \mathrm{Se}$ administró una cefalosporina de primera generación como profilaxis, media hora antes de la incisión cutánea, y heparina de bajo peso molecular, por vía subcutánea, durante 30 días, para la prevención tromboembólica. La rehabilitación consistió en deambular con andador desde el segundo día posterior a la cirugía, para luego utilizar dos bastones canadienses desde el día 3 hasta la tercera semana. A partir de entonces, se continuaba con un solo bastón en la mano contraria a la operada, para finalmente marchar sin asistencia a partir de la sexta semana después de la cirugía.

En el momento del cierre del estudio, se contactó a aquellos pacientes que no habían asistido a un control en los últimos dos años y se les solicitó que concurrieran a una entrevista personal. Si no era posible, completaron la entrevista vía telefónica. Asimismo, se les pidió que enviaran un control radiográfico.

Los controles consistieron en un examen físico y uno radiográfico (proyección anteroposterior y lateral) a las 3 y 6 semanas, a los 3 y 6 meses del procedimiento, y luego una visita anual, de no mediar complicaciones.

Las variables analizadas para caracterizar la población fueron: sexo, edad, diagnóstico, tipo de fémur según la clasificación de Dorr, ${ }^{13}$ bilateralidad, tipo de cotilo utilizado (Duraloc ${ }^{\circledR}$ y Pinnacle $®$, Depuy Synthes, Warsaw, Ind., EE.UU.) y par de fricción utilizado (cerámica-polietileno, cerámica-cerámica, metal-metal).

Para el análisis clínico se utilizó el Harris Hip Score ${ }^{14}$ que contempla el nivel de dolor, la marcha, el rango de movilidad y la funcionalidad (desempeño en actividades de la vida cotidiana).

Todas las mediciones estuvieron a cargo de dos evaluadores en forma independiente y, si no se lograba un consenso, eran revisadas por un autor sénior. Comparando la primera radiografía luego de la cirugía con la del último control de mejor calidad, se estudiaron los siguientes aspectos:

1. Osteointegración del tallo: determinada evaluando la condensación endóstica, definida como la formación de nuevo hueso en la interfase implante-hueso; la fijación se clasificó como ósea estable, fibrosa estable o inestable, de acuerdo con los criterios de Engh y cols. ${ }^{15}$

2. Hundimiento: medido a través de la distancia entre el ángulo proximal del trocánter menor y el hombro lateral del tallo femoral $; 9$ se consideró significativo si medía $>2 \mathrm{~mm}$.

3. Aparición de osteólisis femoral proximal (stress shielding).

4. Formación de hipertrofia cortical perióstica: definida como el aumento en el diámetro diafisario femoral alrededor del implante.

5. Evidencia de aflojamiento alrededor de la prótesis: caracterizada por radiolucidez alrededor del tallo, con cavitaciones de más de $5 \mathrm{~mm}$ de largo en las diferentes zonas del fémur proximal, descritas por Gruen y cols. ${ }^{16}$

El análisis de la supervivencia contempló la necesidad de cirugía de revisión por cualquier causa, y se discriminó el requerimiento de revisión por causa femoral.

\section{Análisis estadístico}

Las variables categóricas se describen como frecuencias y porcentajes, mientras que las continuas se expresan como media y desviación estándar. Se empleó la prueba t de Student para analizar las variables numéricas y comparar los valores preoperatorios y posoperatorios. Las variables descriptivas fueron analizadas con la prueba de la ji al cuadrdo o la prueba exacta de Fisher. Se consideró estadísticamente significativo un valor $\mathrm{p}<0,05$. Todos los cálculos se realizaron con el programa Graph Pad Prism 8.0. 


\section{RESULTADOS}

Se excluyó a 150 casos, porque tenían una adecuada alineación en el plano coronal y a tres por no cumplir con el seguimiento mínimo y la imposibilidad de localizarlos por vía telefónica. La serie quedó conformada por 67 RTC en 58 pacientes (6 RTC bilaterales simultáneos y 3 en dos tiempos) con una media de seguimiento de $10.9 \pm$ 0.8 años (rango 10.0-12.7). La incidencia global de los tallos colocados inadecuadamente en el eje coronal fue del 31,8\% (70/220). La descripción de los pacientes se detalla en la Tabla 2.

Tabla 2. Descripción de los pacientes incluidos en el estudio

\begin{tabular}{|c|c|}
\hline Características & $\begin{array}{c}\text { Grupo con deseje } \\
\text { n }(\%)\end{array}$ \\
\hline $\begin{array}{l}\text { Sexo } \\
\text { Masculino } \\
\text { Femenino }\end{array}$ & $\begin{array}{l}38(65,5 \%) \\
20(34,5 \%)\end{array}$ \\
\hline Edad (rango) & $62.8 \pm 8.4(40-71)$ \\
\hline $\begin{array}{l}\text { Lado } \\
\text { Derecho } \\
\text { Izquierdo }\end{array}$ & $\begin{array}{l}32(47,8 \%) \\
35(52,2 \%)\end{array}$ \\
\hline $\begin{array}{l}\text { Diagnóstico } \\
\text { Coxartrosis idiopática } \\
\text { Osteonecrosis } \\
\text { Displasia del desarrollo de la cadera } \\
\text { Coxartrosis por epifisiólisis }\end{array}$ & $\begin{array}{c}58(86,5 \%) \\
5(7,5 \%) \\
3(4,5 \%) \\
1(1,5) \%\end{array}$ \\
\hline $\begin{array}{l}\text { Tipo de fémur }\left(\text { Dorr }^{16}\right) \\
\text { A } \\
\text { B } \\
\text { C }\end{array}$ & $\begin{array}{c}50(74,6 \%) \\
17(25,4 \%) \\
0(0 \%)\end{array}$ \\
\hline $\begin{array}{l}\text { Cotilo } \\
\text { Duraloc } \AA \\
\text { Pinnacle } \AA\end{array}$ & $\begin{array}{l}30(44,8) \% \\
37(55,2 \%)\end{array}$ \\
\hline $\begin{array}{l}\text { Superficie de fricción } \\
\text { Cerámica-Polietileno } \\
\text { Cerámica-Cerámica } \\
\text { Metal-Metal }\end{array}$ & $\begin{array}{c}64(95,5 \%) \\
1(1,5 \%) \\
2(3,0 \%)\end{array}$ \\
\hline
\end{tabular}

\section{Resultados clínicos}

En el último control, todos tenían una buena movilidad de la cadera, marchaban sin asistencia e informaron haber retornado a sus actividades cotidianas y recreativas sin dificultad. Ninguno refirió dolor de muslo luego del RTC. Se observó un incremento estadísticamente significativo del Harris Hip Score al comparar los valores antes de la cirugía y después $(41,05 \pm 6,5$ vs. $90,05 \pm 2,5 ; \mathrm{p}<0,01)$.

\section{Resultados radiográficos}

De los 67 RTC con deseje en el plano coronal, $\geq 2^{\circ}$ en relación con el eje longitudinal de la diáfisis femoral, se observaron $49(73,3 \%)$ caderas en varo con un valor promedio de $2,5^{\circ} \pm 0,6^{\circ}$ (rango $\left.2-4\right)$ y $18(26,7 \%$ ) en valgo, con una media de $2,7^{\circ} \pm 1,2^{\circ}$ (rango $2-7$ ).

Respecto a la integración de los tallos, se detectaron signos radiográficos de osteointegración en 49 (73,1\%) casos y de unión fibrosa estable en 18 (26,9\%) de las caderas operadas. La fijación de los cotilos de la muestra fue satisfactoria en todos los casos.

Se observó osteólisis femoral proximal en dos pacientes (3,0\%): un tallo con un varo de $2^{\circ}$ en el trocánter mayor, con un seguimiento de 11.5 años, y en otra cadera con varo de $3^{\circ}$ en el calcar, con 10.9 años de evolución (Figura 2). En ambos, no hubo complicaciones ulteriores ni evidencia de progresión radiográfica. 


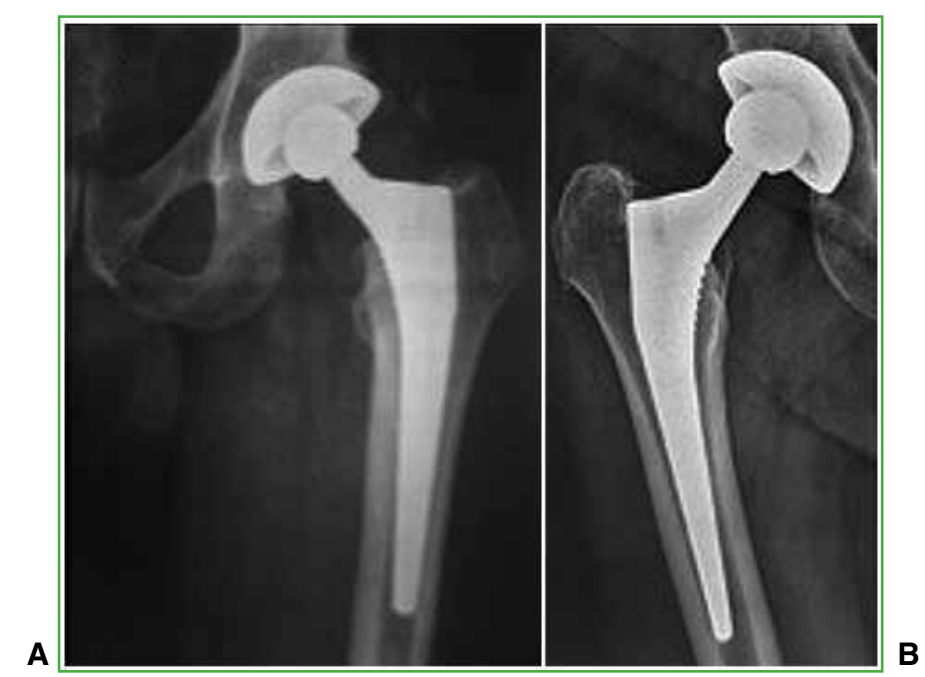

Figura 2. A. Radiografía anteroposterior de un reemplazo total de cadera izquierda, a los 11.5 años de la operación, con tallo en varo. Se observa osteólisis en el trocánter mayor. B. Radiografía anteroposterior de un reemplazo total de cadera derecha a los 11.9 años de la cirugía. Se visualiza osteólisis en el calcar.

No se registraron casos de hundimiento significativo ( $>2 \mathrm{~mm})$, hipertrofia cortical perióstica o radiolucidez alrededor del implante compatibles con aflojamiento.

\section{Complicaciones}

\section{Intraoperatorias}

Se produjeron dos (3\%) fracturas periprotésicas femorales. Al implantar un componente femoral definitivo con $2^{\circ}$ de valgo, se generó una solución de continuidad en el calcar que requirió su extracción, la fijación con doble lazada de alambre y la reimplantación del tallo (Figura 3).

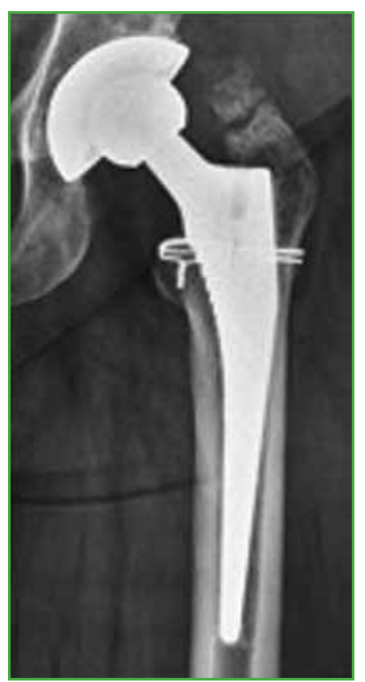

Figura 3. Radiografía de control a los 10 años y 4 meses del reemplazo total de cadera izquierda. Fractura intraoperatoria tratada con lazada de alambre. Tallo con deseje valgo de $2^{\circ}$. Se aprecia también un fragmento del trocánter mayor. 
El paciente evolucionó favorablemente, con buena función y signos radiográficos de estabilidad del tallo, sin hundimiento a los 10 años y 4 meses de seguimiento. El otro caso (valgo de $3^{\circ}$ ) fue una fractura del trocánter mayor no tratada; el paciente tuvo una buena evolución clínica y radiográfica, pese a haber desarrollado seudoartrosis.

Un paciente $(1,7 \%)$ sufrió una isquemia miocárdica intraoperatoria inmediatamente después de la cirugía y requirió intervención urgente a cargo del equipo de cirugía cardiovascular. No se registraron casos de trombosis venosa profunda ni tromboembolismo pulmonar.

\section{Posoperatorias}

Dos pacientes (3\%) presentaron luxación anterior de cadera a los cuatro y seis meses de la cirugía, respectivamente. Uno de los casos fue de origen traumático. Ambos requirieron una reducción incruenta bajo anestesia general, la evolución fue favorable, la complicación no se repitió hasta el último control (10.5 y 12.0 años de seguimiento).

No se detectaron casos de infección de la prótesis o aflojamiento mecánico del tallo femoral.

En el 12\% ( $\mathrm{n}=8$ ) de las artroplastias, se observó un desgaste del polietileno, manifestado por la excentricidad de la cabeza femoral de la prótesis, sin repercusiones clínicas en el último control.

Contemplando la necesidad de cirugía de revisión por cualquier causa, la supervivencia a mediano plazo de los RTC con tallo Corail® inapropiadamente colocados en el plano coronal fue del $100 \%$.

\section{DISCUSIÓN}

Sobre la base de nuestros hallazgos, el incorrecto posicionamiento en el plano coronal de un tallo femoral no cementado recubierto completamente de hidroxiapatita no afectó la supervivencia a largo plazo del implante.

Tradicionalmente, la alineación en varo de los tallos femorales se asocia a malos resultados. ${ }^{17}$ Respecto a los tallos cementados, distintos autores realizaron análisis a largo plazo y comunicaron tasas de falla de entre el 37,5\% y el $46 \%$ cuando se implantan en varo. ${ }^{4,18}$

De manera similar, Gill y cols. ${ }^{19}$ observaron que el posicionamiento en varo del tallo femoral origina un manto delgado de cemento en las corticales proximal medial y distal lateral, generando una concentración de estrés a distal y lateral, que puede causar una fractura del manto de cemento, aflojamiento y hundimiento del componente, así como también fractura periprotésica femoral por insuficiencia lateral.

Desde hace varios años, la fijación no cementada femoral se ha convertido en un procedimiento aceptado globalmente, con buenos resultados a mediano y largo plazo. ${ }^{20}$ El comportamiento de la alineación del tallo femoral en los implantes no cementados varía de acuerdo con el diseño de la prótesis. En las primeras publicaciones, Vresilovic y cols. ${ }^{9}$ obtuvieron resultados desalentadores en tallos no cementados colocados en varo. Observaron que los implantes en mala alineación habían desarrollado una osteointegración defectuosa, con la consiguiente falla en la fijación. En otra publicación de Ries y cols. ${ }^{21}$ sobre el tallo PCA® (Stryker-Howmedica, Mahwah, EE.UU.), la implantación en varo se asoció a un llenado del canal distal insuficiente y a una pobre estabilidad en la punta del tallo. En otro estudio, el grupo de Mallory ${ }^{8}$ analizó una serie de pacientes operados con un tallo de titanio recubierto con plasma spray y hallaron que fue necesario revisar dos implantes femorales por una falla de fijación ocasionada por subdimensionamiento de estos.

La supervivencia de la prótesis de la muestra analizada arrojó un resultado ideal, no hubo cirugías de revisión en el seguimiento a largo plazo. Una posible explicación para los alentadores resultados de nuestra serie podría fundamentarse en el diseño de la prótesis, así como también en el uso de raspas de impactación ósea. En cuanto a la morfología del implante, la porción proximal del tallo Corail® es cuadrangular y ancha, lo que le permite una estabilización tridimensional en el área metafisaria, mientras que la parte distal es cónica, geometría, que le confiere al fémur proximal un gradiente decreciente de rigidez y evita el bloqueo del canal medular distal. ${ }^{22}$

Los resultados de esta serie concuerdan con el trabajo de Schneider y cols. ${ }^{23}$ quienes evaluaron, en un estudio multicéntrico, 3732 RTC con tallo Spotorno ${ }^{\circledR}$ (Zimmer GmbH, Winterthur, Suiza), con un seguimiento promedio de 43 meses. No hallaron una correlación entre la alineación del tallo y la supervivencia de la prótesis, la migración del implante o la aparición de líneas radiolúcidas alrededor de la prótesis. Los autores llegaron a la conclusión de que la alineación en varo del componente femoral no cementado no acarrea consecuencias clínicas ni radiográficas, como sucede con el tallo cementado. Asimismo, Min y cols. ${ }^{24}$ estudiaron la implicancia clínica y de las imágenes de la alineación del tallo femoral. En 98 RTC consecutivos utilizando el mismo implante (Spotorno®) y un seguimiento promedio de 7.7 años, hallaron un $37 \%$ de componentes femorales colocados en varo/valgo en el plano coronal. Compararon los resultados clínicos y radiográficos con los de aquellos RTC con tallo neutro, y no 
obtuvieron diferencias estadísticamente significativas. La fijación obtenida fue la adecuada en todos los casos y la respuesta ósea del fémur proximal fue la misma en los dos grupos. Postulan que la alineación del implante femoral no cementado no compromete la evolución clínica y radiográfica a mediano plazo. En otro estudio retrospectivo de 125 RTC primarios, el grupo de De Beer ${ }^{25}$ identificó 16 casos con alineación en varo (12,8\%). Estudiaron comparativamente los resultados clínicos (Harris Hip Score) y radiográficos (hundimiento y aflojamiento) en 16 RTC con alineación neutra a los cuatro años de evolución, y no encontraron diferencias estadísticamente significativas. Sugieren que, si bien no se recomienda la colocación en varo del tallo femoral, esto no afecta la evolución del RTC a mediano plazo. De manera similar, Khalily y Lester $^{5}$ examinaron 23 RTC con alineación en varo usando un tallo femoral de titanio (Alloclassic $®$, Zimmer GmbH, Winterthur, Suiza), con un mínimo de 5 años de seguimiento. Todos los tallos estaban clínica y radiográficamente estables en el último control. Remarcan que, si bien la alineación en varo puede comprometer los resultados de algunos implantes, el tallo analizado por ellos resultó confiable, a pesar de que había sido colocado en deseje coronal, a los 5-11 años de seguimiento.

Las limitaciones de este estudio son las propias de su carácter retrospectivo y la baja cantidad de casos analizados. Entre las fortalezas, podemos mencionar que se trata de una serie con pacientes tratados en el mismo centro, por el mismo equipo quirúrgico y con una media de seguimiento considerable.

\section{CONCLUSIÓN}

En esta serie, el incorrecto posicionamiento en el plano coronal de un tallo no cementado recubierto completamente de hidroxiapatita no impresionó afectar su comportamiento clínico ni radiográfico a largo plazo. Continuaremos con el seguimiento de estos pacientes a fin de identificar complicaciones potenciales.

Conflicto de intereses: Los autores no declaran conflictos de intereses.

\section{BIBLIOGRAFÍA}

1. Murphy CG, Bonnin MP, Desbiolles AH, Carrillon Y. Varus will have varus; a radiological study to assess and predict varus stem placement in uncemented femoral stems. Hip Int 2016;26(6):554-60. https://doi.org/10.5301/hipint.5000412

2. Vaughan PD, Sing PJ, Teare R, Kucheria R, Singer GC. Femoral stem tip orientation and surgical approach in total hip arthroplasty. Hip Int 2007;17(4):212-7. https://doi.org/10.5301/hip.2008.3618

3. Devitt A, O'Sullivan D, Quinlan W. 16- to 25-year follow-up study of cemented arthroplasty of the hip in patients aged 50 years or younger. J Arthroplasty 1997;12(5):479-89. https://doi.org/10.1016/s0883-5403(97)90169-8

4. Jaffe W, Christopher A, Hawkins A. Normalized and proportionalized cemented femoral stem survivorship at 15 years. J Arthroplasty 1999;14(6):708-13. https://doi.org/10.1016/s0883-5403(99)90226-7

5. Khalily C, Lester DK. Results of a tapered cementless femoral stem implanted in varus. J Arthroplasty 2002;17(4):463-6. https://doi.org/10.1054/arth.2002.32171

6. Sheridan G, Hughes H, Kenny P, Toole GO, Byrne JO. The varus cemented femoral stem in total hip arthroplasty: predictors, implications and the Femoral Access Ratio. J Orthop 2021;23:8-12. https://doi.org/10.1016/j.jor.2020.12.012

7. Iwase T, Morita D, Takemoto G. The effects of patient characteristics and stem alignment on distal femoral cortical hypertrophy after cemented polished tapered stem implantation. Eur J Orthop Surg Traumatol 2020;30(4):559-67. https://doi.org/10.1007/s00590-019-02605-1 
8. Mallory TH, Head WC, Lombardi AV Jr, Emerson RH Jr, Eberle RW, Mitchell MB. Clinical and radiographic outcome of a cementless, titanium, plasma spray-coated total hip arthroplasty femoral component justification for continuance of use. J Arthroplasty 1996;11(6):653-60. https://doi.org/10.1016/s0883-5403(96)80002-7

9. Vresilovic EJ, Hozack W, Rothman RH. Radiographic assessment of cementless femoral components correlation with intraoperative mechanical stability. J Arthroplasty 1994;9(2):137-41. https://doi.org/10.1016/0883-5403(94)90062-0

10. Vidalain J. Twenty-year results of the cementless Corail stem. Int Orthop 2011;35(2):189-94. https://doi.org/10.1007/s00264-010-1117-2

11. Smith S, Garvin K, Jardon O, Kaplan PA. Uncemented total hip arthroplasty: prospective analysis of the tri-lock femoral component. Clin Orthop Relat Res 1991;(269):43-50. PMID: 1864055

12. Bauer R, Kerschbaumer F, Poisel S, Oberthaler W. The transgluteal approach to the hip joint. Arch Orthop Trauma Surg 1979;95(1-2):47-9. https://doi.org/10.1007/BF00379169

13. Dorr LD, Faugere MC, Mackel AM, Gruen TA, Bognar B, Malluche HH. Structural and cellular assessment of bone quality of proximal femur. Bone 1993;14(3):231-42. https://doi.org/10.1016/8756-3282(93)90146-2

14. Aprato A, Jayasekera N, Villar RN. Does the modified Harris Hip Score reflect patient satisfaction after hip arthroscopy? Am J Sport Med 2012;40(11):2557-60. https://doi.org/10.1177/0363546512460650

15. Engh C, Massin P, Suthers K. Roentgenographic assessment. Clin Orthop Relat Res 1990;257:107-28. PMID: 2199114

16. Gruen TA, McNeice GM, Amstutz HC. "Modes of failure" of cemented stem-type femoral components: a radiographic analysis of loosening. Clin Orthop Relat Res 1979;(141):17-27. PMID: 2199114

17. Narayana R, Elbuluk AM, Chen KK, Eftekhary N, Zuckerman JD, Deshmukh AA. Does femoral morphology and stem alignment influence outcomes of cementless total hip arthroplasty with proximally coated double-tapered titanium stems? Hip Int 2020;8:1-8. https://doi.org/1120700019891702

18. Munuera L, Garcia-Cimbrelo E. The femoral component in low-friction arthroplasty after ten years. Clin Orthop Relat Res 1992;(279):163-75. PMID: 1600652

19. Gill J, Sledge JB, Orler R, Ganz R. Lateral insufficiency fractures of the femur caused by osteopenia and varus angulation. A complication of total hip arthroplasty. J Arthroplasty 1999;14(8):982-7. https://doi.org/10.1016/s0883-5403(99)90014-1

20. Jacquot L, Bonnin MP, Machenaud A, Chouteau J, Saffarini M, Vidalain JP. Clinical and radiographic outcomes at 25-30 years of a hip stem fully coated with hydroxylapatite. J Arthroplasty 2018;33(2):482-90. https://doi.org/10.1016/j.arth.2017.09.040

21. Ries MD, Lynch F, Jenkins P, Mick C, Richman J. Varus migration of PCA stems. Orthopedics 1996;19:581-5; discussion 585-6. PMID: 8823815

22. Chatelet J. The cancellous environment: A privileged partner. En: Vidalain J-P, Ait Si Selm T, Beverland D, et al (eds.). The CORAIL® Hip System. Berlin: Springer-Verlag; 2011:34-9.

23. Schneider U, Breusch SJ, Thomsen M, Wirtz DC, Lukoschek M. [Influence of implant position of a hip prosthesis on alignment exemplified by the CLS shaft]. Unfallchirurg 2002;105(1):31-5. [En alemán] https://doi.org/10.1007/s113-002-8162-6

24. Min B, Song K, Bae K. The effect of stem alignment on results of total hip arthroplasty with a cementless taperedwedge femoral component. J Arthroplasty 2008;23(3):418-23. https://doi.org/10.1016/j.arth.2007.04.002

25. de Beer J, Mckenzie S, Hubmann M, Petruccelli D, Winemaker M. Influence of cementless femoral stems inserted in varus on functional outcome in primary total hip arthroplasty. Can J Surg 2006;49(6):407-11. PMID: 17234069 